\title{
IDENTIFICATION AND DURABILITY OF LEAD GlaSS-Filled RuBiES
}

\author{
Shane F. McClure, Christopher P. Smith, Wuyi Wang, and Matthew Hall
}

\begin{abstract}
In early 2004, the GAAJ laboratory in Japan issued a lab alert about rubies they had seen that had large numbers of fractures filled with high-lead-content glass, which made them appear very transparent. Since then, large quantities of this material have reached international markets. This dramatic treatment is not difficult to identify with a standard gemological microscope, since it has characteristics similar to clarity-enhanced diamonds (flash effect, gas bubbles, etc.).

However, locating filled cavities in reflected light is more challenging, as the surface luster of the filler is close to that of ruby. The filling material appears to be very effective in reducing the appearance of fractures. Durability testing of a few samples by highly skilled jewelers indicated that the filler was fairly resistant to heat exposure during jewelry repair procedures, but it reacted readily with solvents.
\end{abstract}

G em corundum has been a mainstay of the jewelry industry for centuries. The demand for rubies and sapphires has usually outdistanced supplies, and for much of history only the very wealthy could afford them. With the discovery of additional deposits during the 20th century, the supply of these gems increased dramatically. However, there continued to be more demand for these beautiful stones than Mother Nature could provide.

Thus enters the art of treatment. We use the term art here because many if not most of the treatments were not developed by scientists but rather by experimenters who relied largely on luck or trial and error. Many of those who developed these techniques never fully understood the science or the "why" of what they were doing, but they understood the "what" and the "how" very well.

Corundum, as a very durable material, lends itself to many treatments. And ruby, being the most prized color of corundum, is often a prime focus of these treatments. Over the years, ruby has been subjected to heat treatment to change its color and/or improve its clarity; fracture healing to improve clarity and get a higher yield from naturally fractured rough; glass filling of cavities to improve appearance and add weight; and diffusion, dyeing, coating, and synthetic overgrowth, among others.

The latest venture into ruby treatments involves an improvement in clarity enhancement. In the past, the fractures in rubies have been filled with oils, which do little to improve apparent clarity, and glasses, mostly silica based, which are better than oils but, in our opinion, still not very effective because of their relatively low refractive index.

This newest treatment is based on the same principle that has been applied to emerald and diamond: use of a filling material that closely matches the refractive index of the host material to minimize the appearance of the fractures. In the case of this new treatment, the results are remarkable (figure 1). This article looks at the introduction of this technique, its identification in ruby, and its response to various durability tests.

See end of article for About the Authors and Acknowledgments. Gems \& Gemology, Vol. 42, No. 1, pp. 22-34.

(C) 2006 Gemological Institute of America 


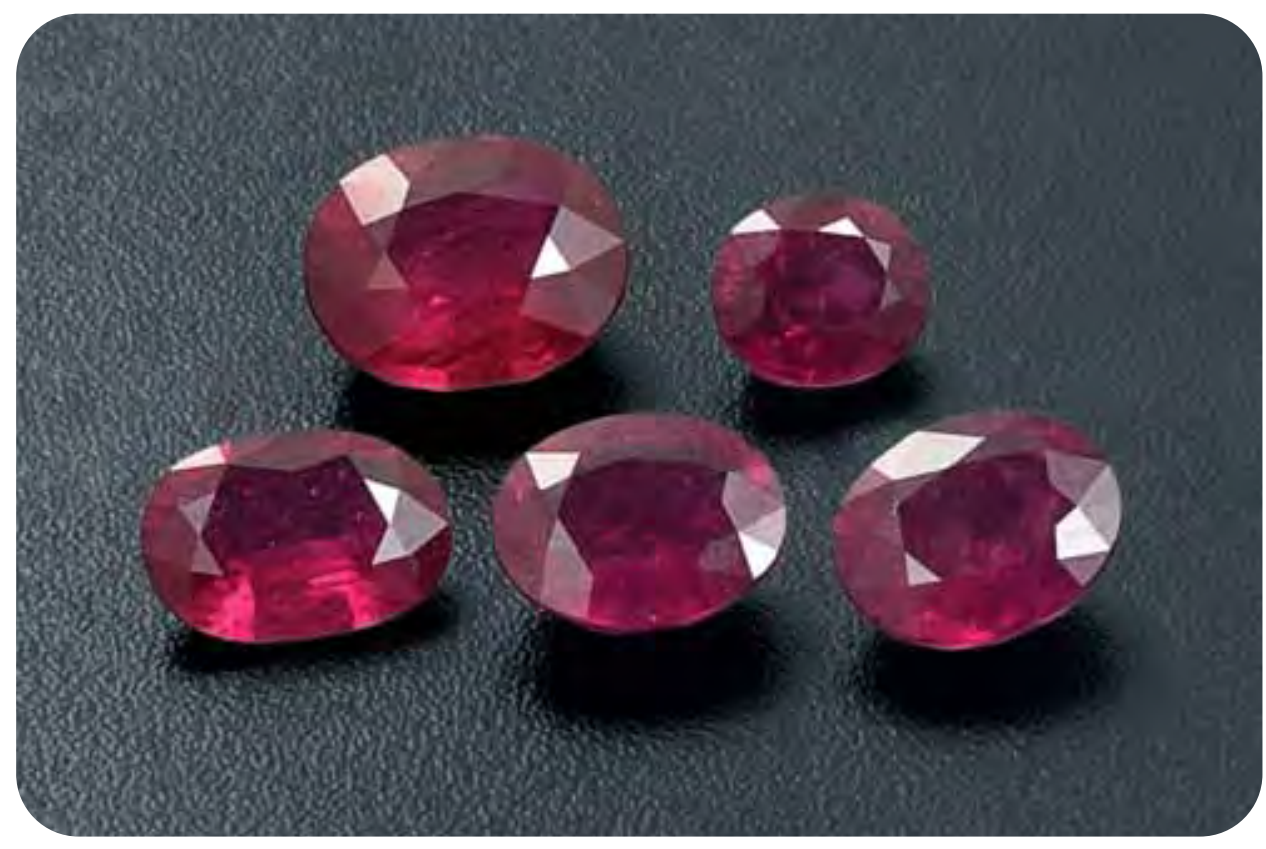

Figure 1. These stones (2.15-7.42 ct) are typical of the final result achieved with the filling of fractures in rubies with high-lead-content glass. Photo by Elizabeth Schrader.

\section{BACKGROUND}

Silica glass has been used extensively to fill cavities and fractures in rubies since the 1980s. Cavity filling was noted first, and it was described as early as 1984 (Kane, 1984). This filling did improve the stones' face-up appearance and could add weight, but it could also readily be detected with magnification.

The early 1990s witnessed the marketing of huge quantities of ruby from Mong Hsu, Myanmar, with multiple cavities and fractures that were filled with, or partially healed by, glassy substances added during high-temperature heat treatment (Peretti et al., 1995; McClure and Smith, 2000). The term residue began to be applied to this kind of material, in reference to the glass that was a side effect of the real intent, which was to heal the fractures. The R.I. of this silica glass is significantly lower than that of the host corundum, so even a fracture entirely filled with it can still be easily seen (figure 2). Therefore, even though the appearance of the fractures is improved, silica glass is not the most efficient material for enhancing the clarity of rubies.

The first report of a new type of ruby clarity treatment came in an Internet alert issued by the Gemmological Association of All Japan in early 2004 (GAAJ Research Laboratory, 2004). They described rubies with inordinate amounts of very low-relief fractures that had been filled with a high-lead-content glass. Since the GAAJ report, a large number of these stones have been examined by gemological laboratories around the world, and they have been offered for sale at trade shows in Bangkok, Hong
Kong, Switzerland, the United States, and elsewhere. Rubies below $1 \mathrm{ct}$ to over $100 \mathrm{ct}$ have been identified as lead-glass filled (see, e.g., figure 3), with a large number between 5 and $10 \mathrm{ct}$. In addition to the GAAJ lab alert, several articles have provided observations on this material (see, e.g., AGTA, 2004, 2005, 2006; Rockwell and Breeding, 2004; Li-Jian et al., 2005; Milisenda et al., 2005; Pardieu, 2005; Smith et al., 2005; SSEF, 2005; Sturman, 2005; Themelis, 2005).

Lead-Glass Filling of Rubies. The actual treatment process was described by Vincent Pardieu of the

Figure 2. Silica glass (in the older method) has a significantly lower R.I. than corundum. When it is used to fill fractures in ruby, it improves their appearance, but the fractures are still very visible. Photomicrograph by S. F. McClure; magnified 30x.

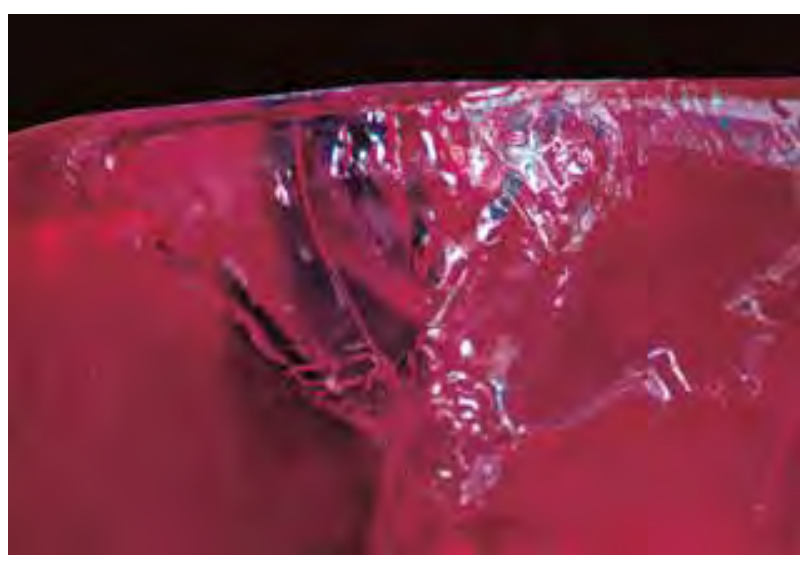




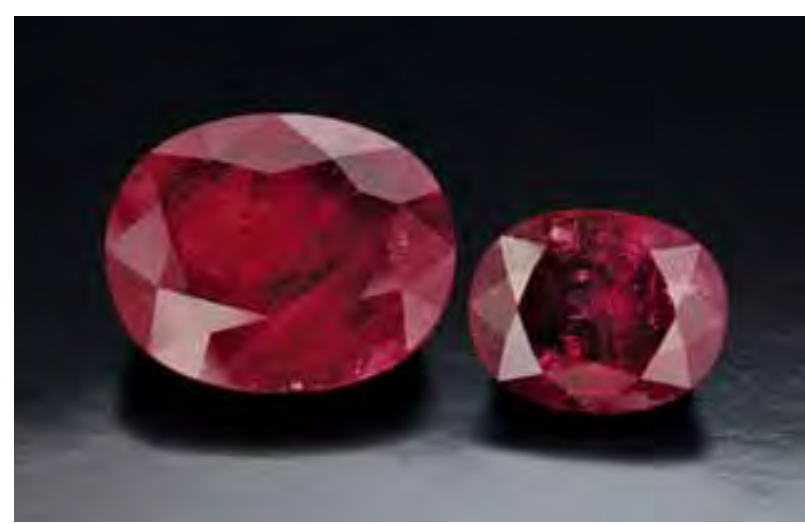

Figure 3. Many extremely large lead glass-filled rubies (here, 52.60 and $26.07 \mathrm{ct}$ ) have been seen in the market. Rubies courtesy of Golden Stone USA Inc., Los Angeles; photo (C) Robert Weldon and GIA.

Asian Institute of Gemological Sciences (AIGS; Pardieu, 2005). The following description is summarized from that article. Mr. Pardieu cited as the source of this information the person purported to be doing the treatment at the time, Mahiton Thondisuk of Chantaburi, Thailand.

The first step involves preforming the material to remove any matrix or obvious impurities. The second step is referred to as "warming," that is, heating the stone to moderate temperatures (reportedly $900-1,400^{\circ} \mathrm{C}$ ). Often used as a first step in standard heat treatment, "warming" removes potential impurities from the fractures and may improve the color.

The third step involves mixing the stone with powders that are composed primarily of lead and silica but may also contain sodium, calcium, potassium, and metal oxides such as copper or bismuth. This mixture is then heated again, reportedly to approximately $900^{\circ} \mathrm{C}$, fusing the powders into a glass that penetrates the fractures in the stone.

Unlike the process that produces the silica-glass fillings seen previously, the filling of corundum with lead glass initially did not involve the partial healing of fractures. In fact, there was significant evidence to show that these stones had not been exposed to the high temperatures necessary to heal fractures.

According to Pardieu, the original starting material for lead-glass filling was very low grade pink, red, or purplish red corundum from Andilamena in Madagascar that was typically translucent to opaque. For the most part, it is unusable as a gem in its natural state (figure 4). Of course, this treatment can be applied to fractured ruby from any locality. We have now seen lead glass-filled stones that appeared to be from Tanzania and Myanmar. The effectiveness of the treatment is amazing, in that it transforms corundum that is opaque and nearly worthless into material that is transparent enough for use in jewelry.

The Present Study. To characterize this treated material as well as determine its identifying features, we examined dozens of samples by standard gemological methods and other analytical techniques. We also tested the durability of the treatment by subjecting samples to routine jewelry manufacturing and repair procedures, as well as to conditions of standard wear and care. The results of this testing, and procedures for identification, are described here.

Readers should bear in mind that the properties we report below are restricted to those observed in the samples we obtained for this study. Although a broad range of samples were selected from several different vendors over more than 14 months, stones treated in a similar fashion but with glass of a different composition may be in the market, and these may have different properties and different reactions to the durability tests.

\section{MATERIALS AND METHODS}

We collected the samples of lead glass-filled rubies and pink sapphires used for this study from late 2004, when large quantities of this treated material first became available on the market, until February 2006. We obtained them in Bangkok and New York City in late 2004, at the June 2005 JCK and AGTA Las Vegas gem shows, and at the 2005 and 2006 Tucson gem shows, all from different sources. We examined a total of 50 faceted samples, ranging from 0.43 to 9.19 ct.

Standard gemological equipment was used to characterize the basic properties of 10 selected samples including: a refractometer, a desk-model spectroscope, long- and short-wave ultraviolet lamps, and a polariscope. All samples were examined with a binocular microscope and fiber-optic illumination.

Qualitative (30 samples) and semiquantitative (2 samples) chemical analyses were performed by energy-dispersive X-ray fluorescence (EDXRF) spectroscopy using a Kevex Omicron spectrometer operated at a voltage of $25 \mathrm{kV}$ with no filter, a 50 micron collimator, and a 500 second livetime.

Observations and chemical analyses were also performed on four samples using a JEOL-JXA8800 scanning electron microscope with a wavelengthdispersive spectrometer (SEM-WDS) at the Geophysical Laboratory of the Carnegie Institution of 
Washington, in Washington, DC. Operating conditions for both electronic imaging and wavelengthdispersive analyses were $10 \mu \mathrm{A}$ beam current and 15 $\mathrm{kV}$ accelerating potential. The presence of any element with a concentration above $100 \mathrm{ppm}$ (from B to $\mathrm{U}$ in the periodic table) will be detected. Even though we used a focused electron beam, which was about $1-2 \mu \mathrm{m}$ in diameter, due to the limited surface area of the filling material and the poor quality of the polish on most of the samples tested, chemical analysis was performed without calibration against standard materials.

X-radiography was performed on five samples using a Hewlett-Packard Faxitron series X-ray cabinet.

The samples were also tested for their durability in standard conditions of manufacture, wear, and repair. Heating experiments were performed on a total of 10 samples using a Lindberg/Blue box furnace in an ambient atmospheric environment. The temperature of the furnace was raised to the target values first and then the sample (held in an $\mathrm{Al}_{2} \mathrm{O}_{3}$ ceramic disk) was placed inside. The temperatures were 100, $200,600,700,800$, and $1,000^{\circ} \mathrm{C}$. Selected samples were exposed to each of these temperatures for periods of 5,10 , and 60 minutes each. In addition, one sample was held at $200^{\circ} \mathrm{C}$ for an extended period of 16 hours. After a specific heating period, the samples were taken out, cooled in air, and reexamined.

We also exposed eight rubies filled with high-leadcontent glass to a series of jewelry repair procedures. These included steam cleaning, ultrasonic cleaning, setting (including mounting, filing and polishing), and retipping of prongs. Details on these tests are given in the section on durability testing below.

A total of eight reagents were used to assess the $\mathrm{Pb}$-glass filler's resistance to chemical attack. Three reagents consisted of caustic soda, aqua regia (nitrohydrochloric acid), and a standard jeweler's pickling solution (sodium bisulphate). The latter two are frequently used in jewelry manufacturing or repair; caustic soda is a more reactive base than standard pickling solution. We also tested the treatment for its durability to a range of household products: concentrated lemon juice, a typical aerosol oven cleaner, ammonia, a standard drain cleaner, and bleach. For each chemical (with the exception of the pickling solution, for which three samples were used), one ruby with $\mathrm{Pb}$-glass filler was placed in a beaker and covered with the reagent (typically 10-20 ml). In addition to using a fume hood for all experiments, we covered toxic solutions (aqua regia and ammonia) with a baking soda filter over the beaker top to min-

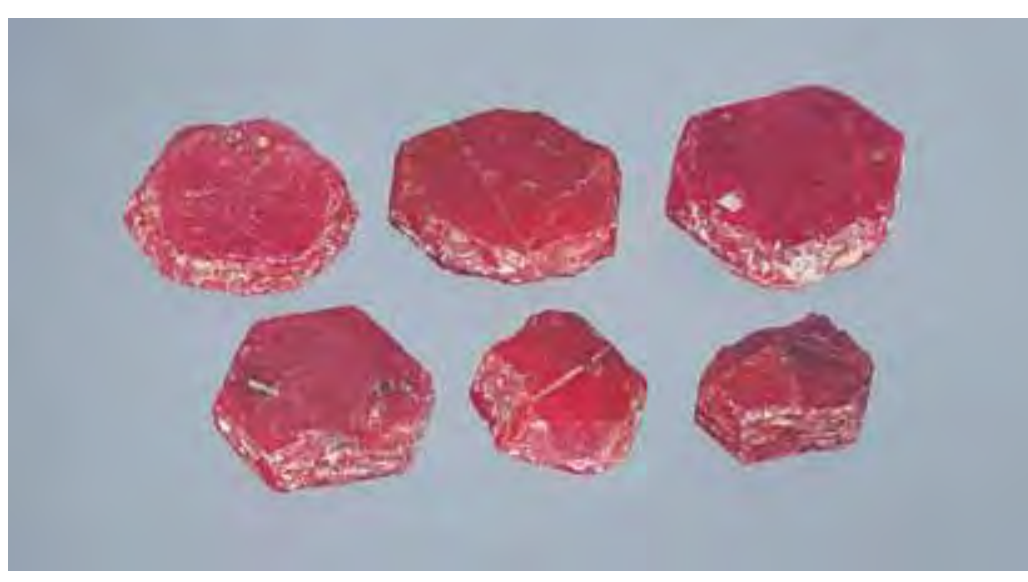

Figure 4. The starting material for this ruby treatment is very low quality and until now was only useful as mineral specimens. The crystals shown here range from 7.28 to 22.08 ct. Photo by Maha Calderon.

imize noxious fumes. Each experiment run was conducted at or just below the boiling point to accelerate any reaction; a small laboratory hot plate with variable temperature control was constantly adjusted to keep the reagents at this temperature. Experiment run time was four hours (except for aqua regia, which was one hour long), to mimic the cumulative effect of multiple exposures for shorter periods of time. The beaker was then removed from heat and allowed to cool. Once cooled, the stones were cleaned and examined for alterations to the Pb-glass filler.

\section{RESULTS}

Visual Appearance. All the samples collected for this study were transparent to semitransparent and could be considered jewelry quality. The color of many of the specimens was slightly brownish and often of lower saturation-so that some of them would be considered pink sapphire. However, several of the authors saw large parcels of lead glass-filled corundum in early 2006 that could easily be categorized as medium-quality ruby. Two of these rubies were acquired for this study (one is shown in figure 5).

Standard Gemological Properties. The long- and short-wave UV fluorescence, visible-range absorption spectrum, pleochroism, birefringence, optic character, and specific gravity were consistent with ruby/pink sapphire in general. It is interesting to note, however, that the specific gravity of one 1.34 ct stone was slightly higher (4.03) than usual for corundum. This stone had several large, deep, filled cavities. We do not have data on the S.G. of the glass filler, but it is well 


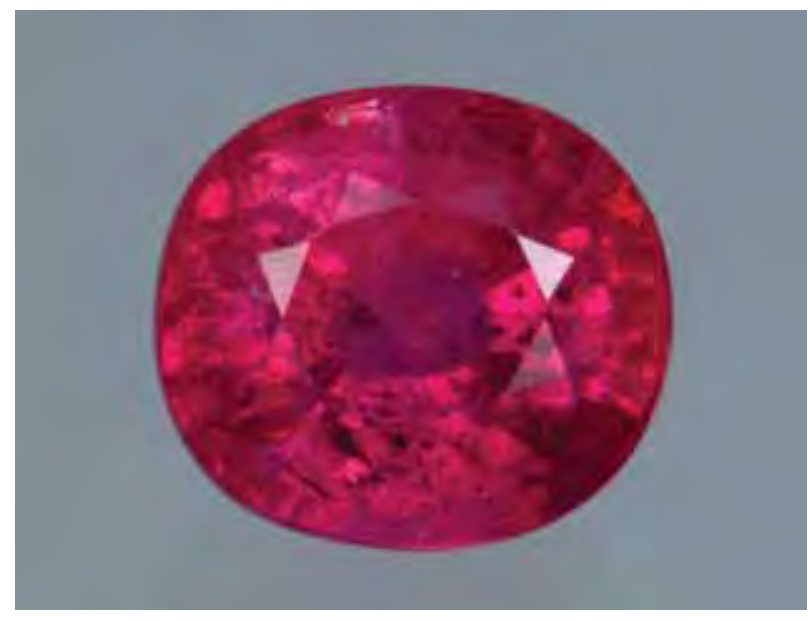

Figure 5. Some of the specimens we acquired for this study, such as this $2.85 \mathrm{ct}$ stone, would be considered medium-quality ruby. Photo by C. D. Mengason.

known that high-lead-content glass has a high specific gravity, so it is not surprising that it could affect the overall S.G. of the stone. Standard R.I. readings and birefringence were recorded for all of the samples. In addition, we obtained a single approximate R.I. reading of 1.75-1.76 for the lead-glass filler on areas where it filled larger cavities.

Internal Features. All the samples we examined revealed naturally occurring internal features that ranged from extensive twinning and parting planes to various mineral inclusions. Some mineral inclusions

Figure 7. One of the most important identification features of this treatment is a blue flash effect similar to that seen in filled diamonds and emeralds. The strength of the flash varied considerably, sometimes being relatively weak, as can be seen in this example. Photomicrograph by S. F. McClure; magnified 15x.

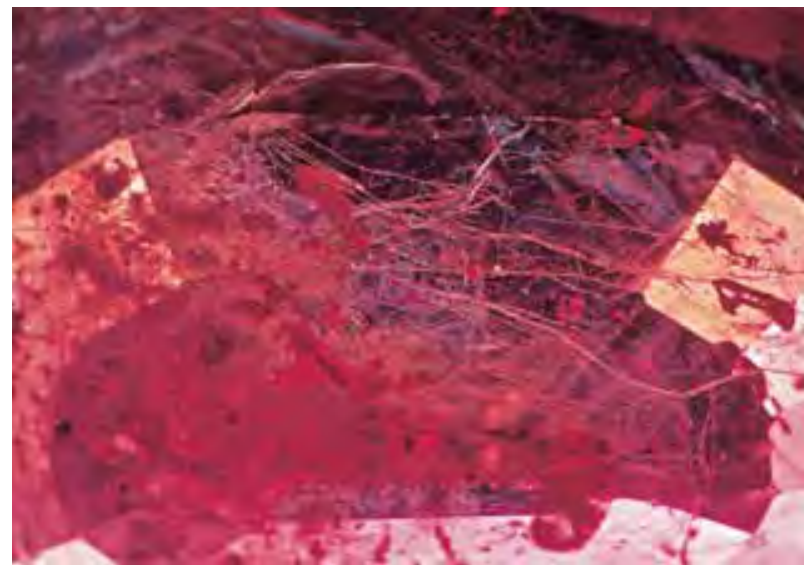

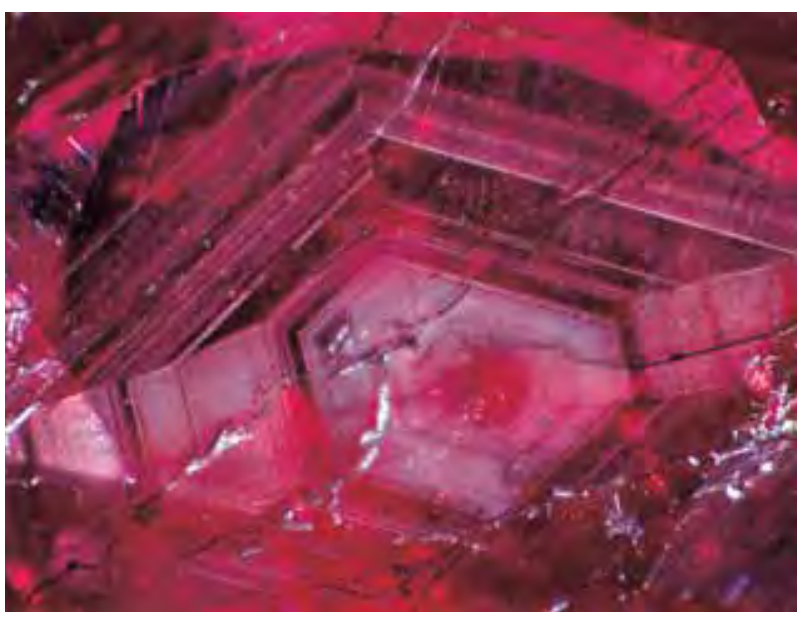

Figure 6. Dense clouds of fine, unaltered rutile needles following the growth structure prove that this lead glass-filled ruby has not been exposed to extremely high temperatures. Photomicrograph by S. F. McClure; magnified 20x.

showed evidence of thermal alteration, whereas others did not. Most significantly, many of these stones revealed dense clouds of fine, unaltered rutile needles following the hexagonal structure of the ruby (figure 6). This is clear evidence that these stones had not been exposed to temperatures high enough to damage rutile (greater than $1,500^{\circ} \mathrm{C}$; Emmett et al., 2003).

When examined with a microscope or a standard jeweler's loupe, virtually all the samples were dominated by numerous large fractures of very low relief. In addition, blue flashes were readily noted as the

Figure 8. In some of the samples, the flash effect was very strong and an orange flash could also be seen. Here, the blue is quite strong in brightfield illumination, and the orange flash is easily seen in darkfield. Photomicrograph by S. F. McClure; magnified 10x.

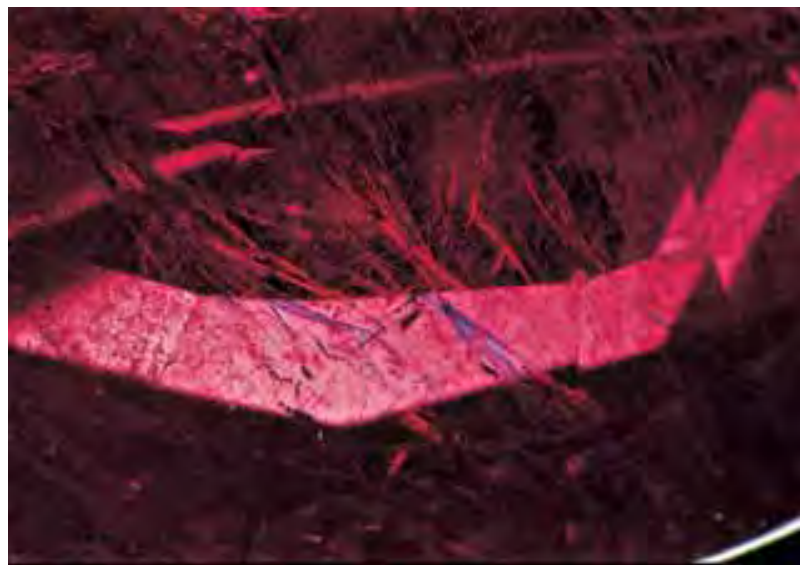



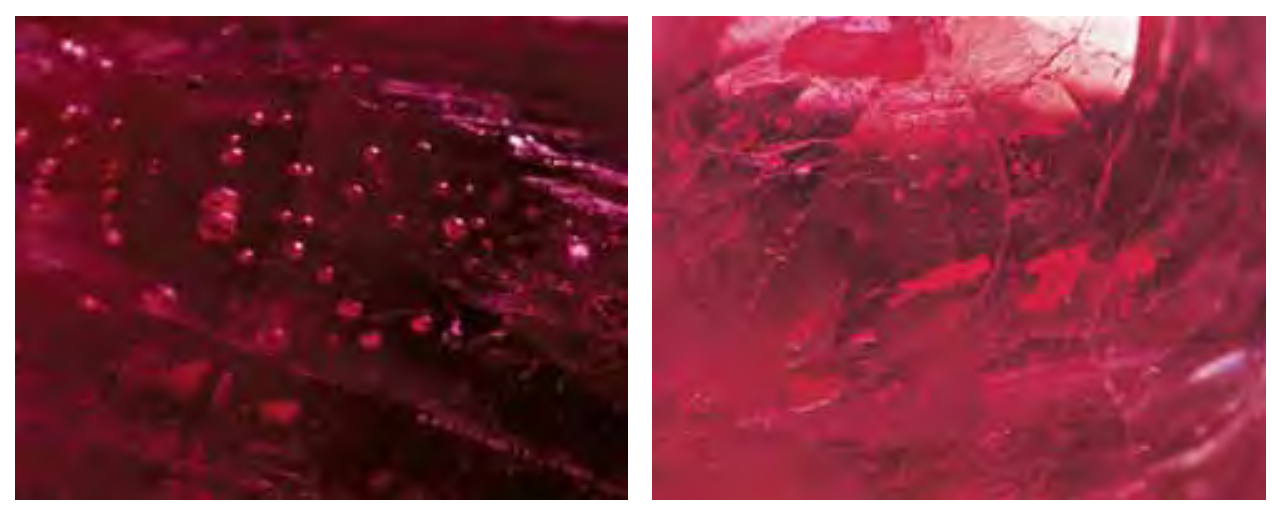

Figure 9. Numerous

flattened and rounded gas bubbles were present in almost all the corundum with filled fractures that we examined. Photomicrographs by C. P. Smith (left, $28 \times)$ and S. F. McClure (right, 20×). stones were rotated and repositioned for a complete view of the interior. The strength of the flash effect varied from relatively subtle (figure 7) to quite strong, although usually with the same intensity in samples obtained from the same source. In some stones, an orange flash was visible as well (figure 8). Also seen with magnification in all the samples were numerous flattened and rounded gas bubbles and voids within the glass fillings (figure 9). Where filled cavities were large enough, spherical gas bubbles were sometimes visible. This was reminiscent of the features we first noted in clarity-enhanced diamonds 18 years ago (Koivula et al., 1989). Although the filling material in rubies might be different, the effect of the treatment-to minimize the visibility of fractures and cavities-was almost identical to that achieved with fracture filling in diamonds.

In the majority of the samples, the glass filling did not appear to be colored. However, we did note that along thick seams or cavities of the glass there was a distinct yellow hue in a few samples and a more subtle pink coloration in others. In several of the lower-

Figure 10. The yellow color of the lead-glass filler is visible here in a very large internal cavity.

Photomicrograph by S. F. McClure; magnified 30x.

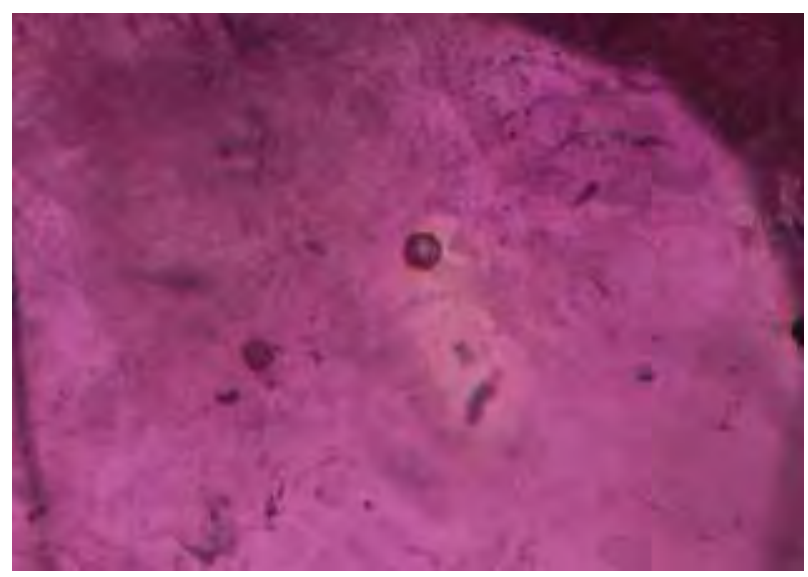

quality samples we acquired at the Tucson shows in 2006, we observed large filled cavities where the yellow color of the filler was readily apparent, even through the body of the stone (figure 10).

Surface Characteristics. Previously, examining the surface of some heated rubies in reflected light would reveal the presence of cavities or depressions that had become a reservoir for the flux typically used to induce the healing of fractures. These agents often would form a silicon-rich glass that had a significantly lower refractive index than the ruby host, resulting in a lower surface luster. We were somewhat surprised to see how effective this new treatment was at reducing the surface visibility of large cavities and wide fractures, which in some cases extended across the width or length of the sample. In many of the study samples, we noted that lead glass-filled cavities, even very large ones, were difficult to detect. Cavities filled with silica glass or heating residues are typically very visible - even with darkfield illumination (figure 11). Use of the higher-R.I. lead glass, how-

Figure 11. Cavities filled with silica glass typically remain very visible in the microscope. Photomicrograph by S. F. McClure; magnified 37x.

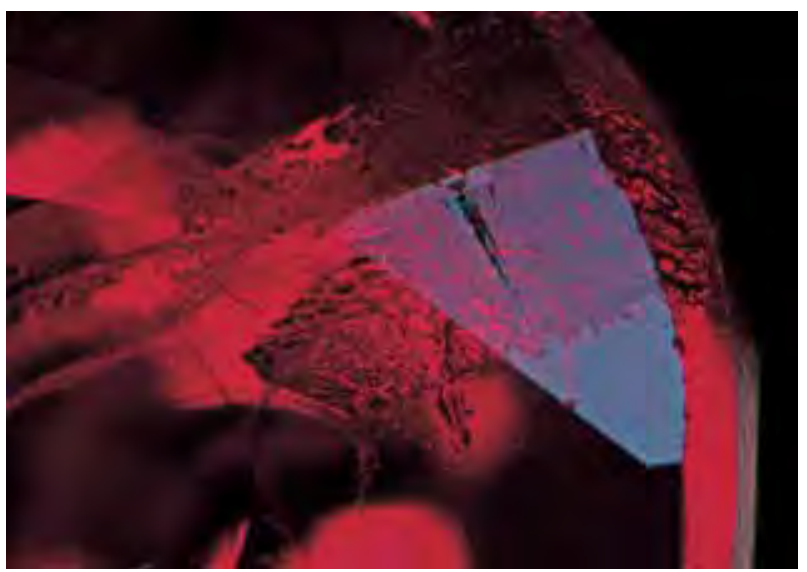



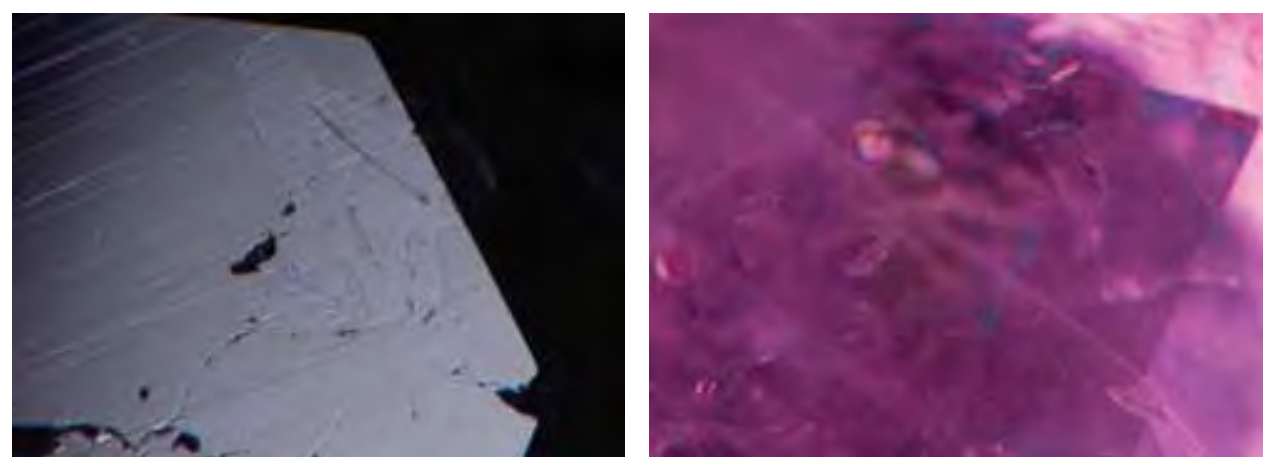

Figure 12. Cavities filled with high-lead glass can be challenging to see. In the image on the left (reflected light), the surface luster of the filler is very close to that of the corundum. On the right, the same filled cavity is not visible at all in darkfield; only the outline of blue flash suggests its presence. Photomicrographs by S. F. McClure; magnified 30x.

ever, makes the cavities virtually disappear (figure 12). Even the traditional reflected-light technique was less reliable, as careful positioning of the stone in figure 12 was necessary to make the subtle difference in surface luster visible. Many examples were seen where the surface luster of the glass was comparable to the luster of the ruby, and only careful examination revealed that the luster was lower than, equal to, or higher than (figure 13) that of the ruby. Many times the only noticeable difference was in the quality of the polish: Glass, particularly high-lead-content glass, is significantly softer than corundum, which makes the polish noticeably inferior to the host (figure 14). In a few samples, we also noted that shrinkage had occurred in the Pb-glass that was filling cavities (figure 15).

It is interesting to note that one lead glass-filled ruby that was submitted to the GIA Laboratory showed evidence of oxidation of the filler at the surface, undoubtedly a consequence of the extreme lead content (figure 16).

Figure 13. Sometimes the surface luster of the leadglass filling is noticeably higher than the surrounding corundum. Photomicrograph by S. F. McClure; magnified 36x.

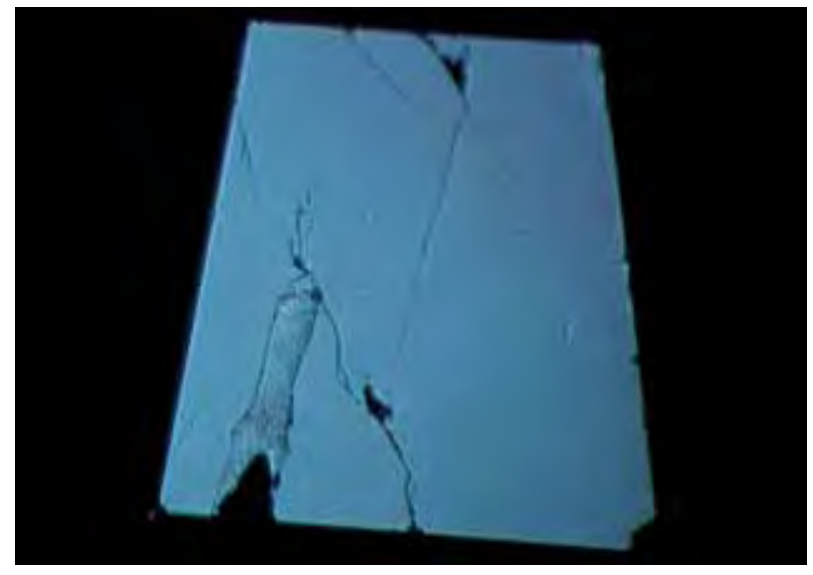

Chemical Composition. All 30 of the samples that were analyzed qualitatively with EDXRF showed a significant lead content, as did the two measured semiquantitatively. To obtain a more precise evaluation of the composition of the glass filler alone, we analyzed fillings in four stones by SEM-WDS.

SEM is a very useful technique for analyzing lead glass-filled ruby, because the glass and surrounding ruby show a large difference in brightness in backscattered-electron (BSE) images (figure 17). The filling process is so efficient that the glass can successfully penetrate fractures as thin as $5 \mu \mathrm{m}$. BSE images taken at high magnification illustrate that the boundary between the glass and the host ruby is sharp. No precipitation of secondary corundum was observed in any of the samples analyzed. In contrast, in some Si-rich glass-filled rubies we have examined in the lab, we observed that the deposition of secondary corundum formed a zigzag boundary between the glass and the host ruby (figure 18).

SEM-WDS chemical analysis of glass in fractures

Figure 14. Glass, particularly high-lead-content glass, is significantly softer than corundum, so sometimes the best way to notice a glass-filled cavity in reflected light is by the poor polish on its surface.

Photomicrograph by C. P. Smith; magnified $45 \times$.

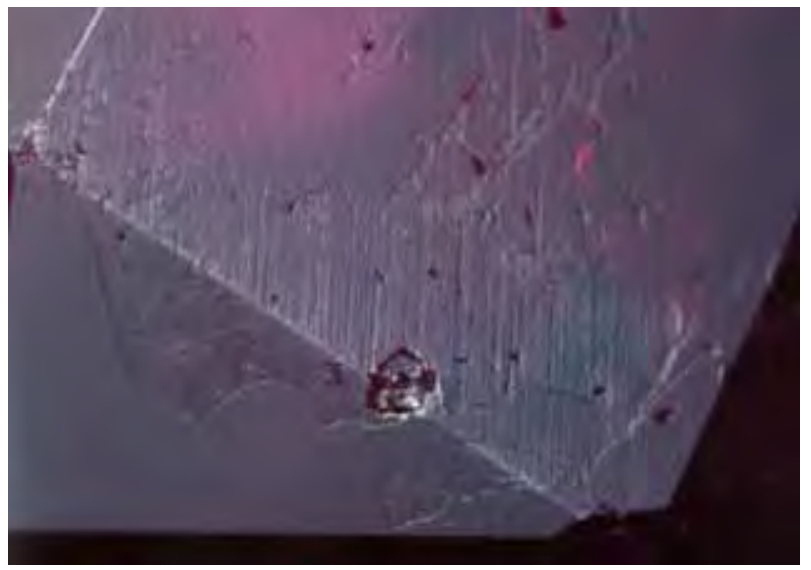




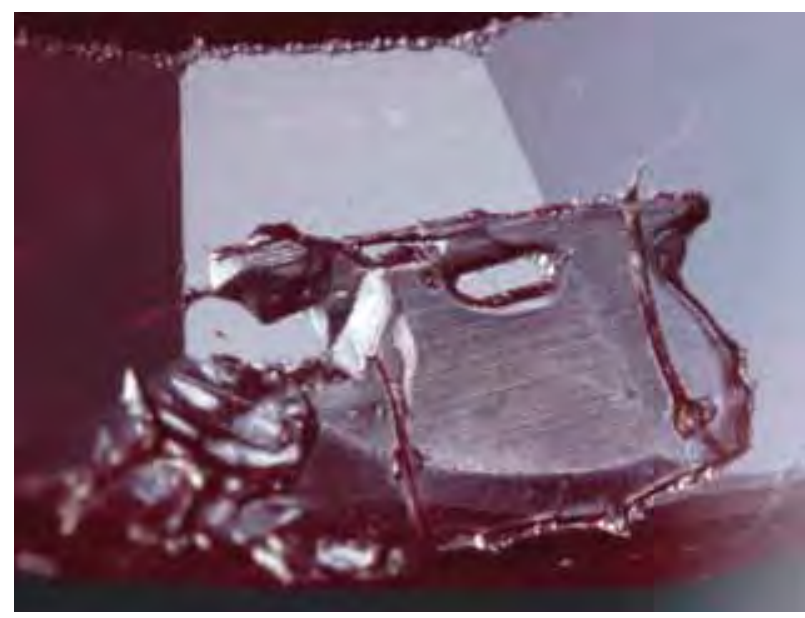

Figure 15. Shrinkage of the glass in this large cavity (over $1.0 \mathrm{~mm}$ in longest dimension) appears to have taken place subsequent to polishing, causing the polished surface of the glass to be lower than that of the surrounding ruby. Photomicrograph by C. P. Smith; magnified $45 x$.

less than $10 \mu \mathrm{m}$ wide is problematic due to beam overlap with the surrounding ruby. However, consistent results were obtained for glass-filled areas with relatively large surfaces: major components$\mathrm{PbO}\left(71-76\right.$ wt.\%), $\mathrm{Al}_{2} \mathrm{O}_{3}(12-15$ wt. $\%)$, and $\mathrm{SiO}_{2}$ (11-13 wt.\%); minor components $(<1$ wt.\%)$\mathrm{Na}_{2} \mathrm{O}, \mathrm{K}_{2} \mathrm{O}, \mathrm{CaO}, \mathrm{FeO}$; and trace amounts of $\mathrm{MgO}$, $\mathrm{P}_{2} \mathrm{O}_{5}$, and $\mathrm{TiO}_{2}$. These results are similar to those that have been described by others (e.g., Li-jian et al., 2005).

Figure 17. This back-scattered-electron image (taken with a scanning electron microscope) shows lead glass-filled fractures of varying widths. Because of the higher average mass of elements in the glass, it is much brighter than the host ruby. Width of the image is about $1.6 \mathrm{~mm}$.

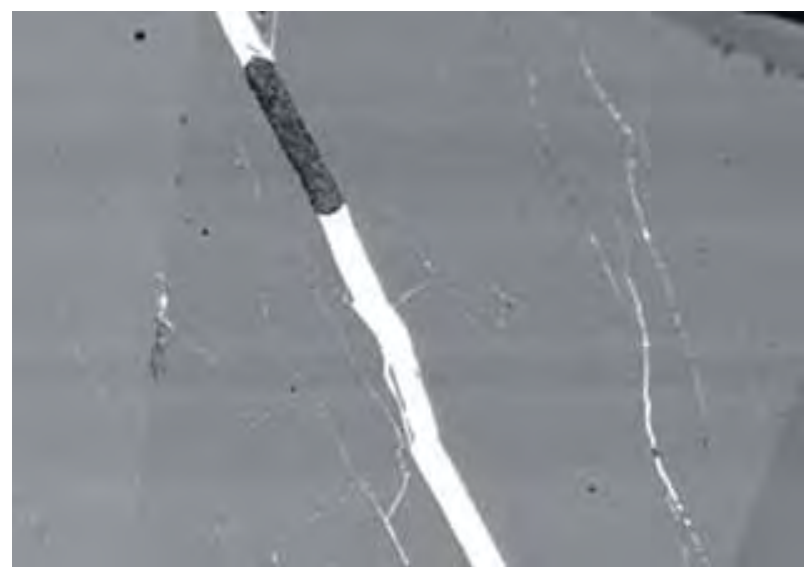

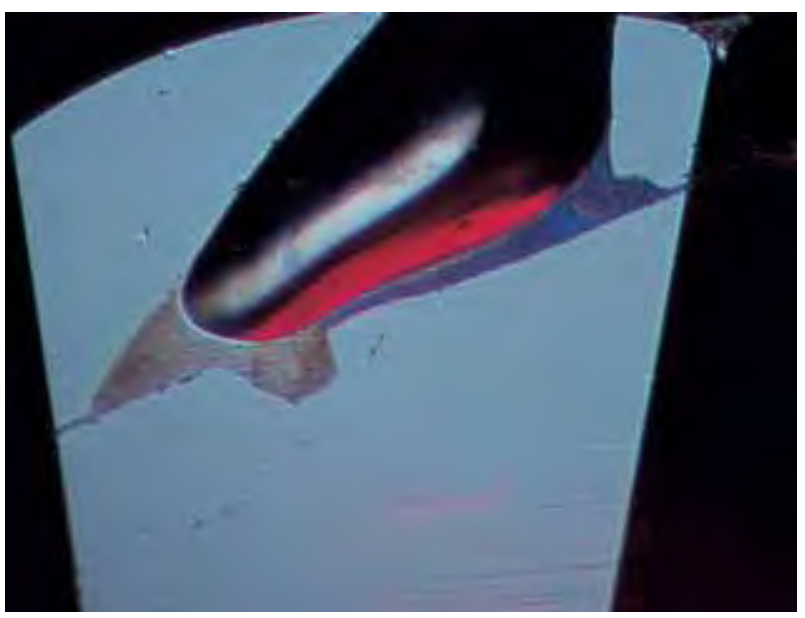

Figure 16. One example recently submitted to the GIA Laboratory showed an obvious oxidization of the glass at the surface. Photomicrograph by S. F. McClure; magnified 40x.

It is interesting to note that semiquantitative EDXRF analyses of the lead glass in two samples acquired in 2006 showed very similar composition to that of rubies acquired in 2004, which suggests that the composition is fairly consistent over time.

$\mathbf{X}$-radiography. This test was shown to be successful in detecting the high-lead-content glass used for filling fractures in diamonds many years ago (Koivula et al., 1989), working on the basis that lead glass strongly absorbs X-rays. As expected, the lead glass-filled fractures in our samples were easily visible in the Xradiograph (figure 19). Others have also reported using this test effectively (Befi and Dutoit, 2005; Kitawaki et al., 2005).

\section{DURABILITY TESTING}

Once a method of detection for any given treatment is established, invariably the next question that arises is "Is it durable?" To test this, we set up a series of experiments, focusing mainly on the conditions these stones might encounter in normal situations of wear, care, and manufacture or repair. We first reported the results of some of these tests at the 2005 ICA Congress in Bangkok, Thailand.

Controlled Heating. The first question we wanted to answer was how much heat this lead-glass filler could survive. The melting point of the material is very important, as it determines how well the filler can stand up to jewelry manufacturing techniques. We suspected that the glass might not withstand 

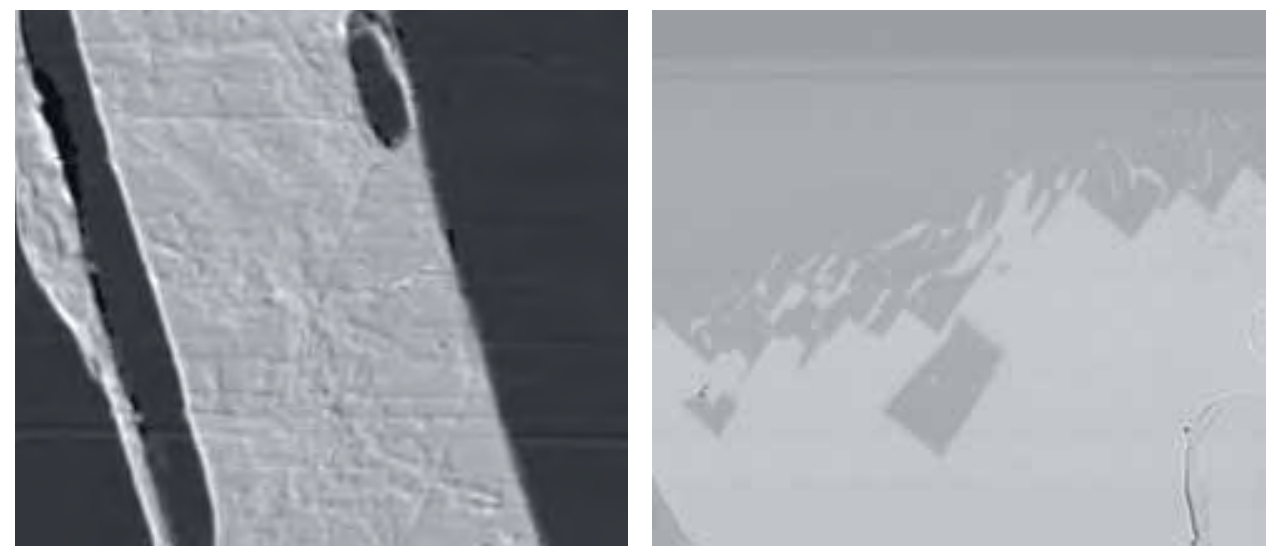

Figure 18. No chemical reaction between the lead glass and the host ruby was detected with the SEM (left, BSE image width 100 $\mu \mathrm{m}$ ); the boundary between the two phases is sharp. In contrast, in the silicon glassfilled ruby (right, BSE image width $390 \mu \mathrm{m}$ ), deposition of secondary corundum formed a zigzag boundary between the glass and the ruby. normal jewelry repair techniques such as the retipping of prongs, primarily because of our experiences with the lead glasses used to fill fractures in diamonds (e.g., Koivula et al., 1989).

No change was observed in the samples held at $100^{\circ} \mathrm{C}$, at $200^{\circ} \mathrm{C}$ for 16 hours, or at $600^{\circ} \mathrm{C}$. At $700^{\circ} \mathrm{C}$, we observed the first sign of a change to the glass: Almost immediately, minor amounts of glass started sweating out of filled fractures and beading on the surface (figure 20). After only a few minutes at this temperature, the glass in larger filled cavities started to melt and flow (figure 21). At $800^{\circ} \mathrm{C}$, the glass started bubbling at the surface of the fractures (figure 22). These tests clearly show that the melting point of the lead glass in these samples is between $600^{\circ}$ and $700^{\circ} \mathrm{C}$. Of course, changes in the composition of the glass from one treater to another or from one time frame to another could result in a change to the melting point.

Figure 19. The lead-glass filler is readily seen in an Xradiograph because lead glass strongly absorbs $X$ rays. Some scattered zircon crystals are also visible in this image.

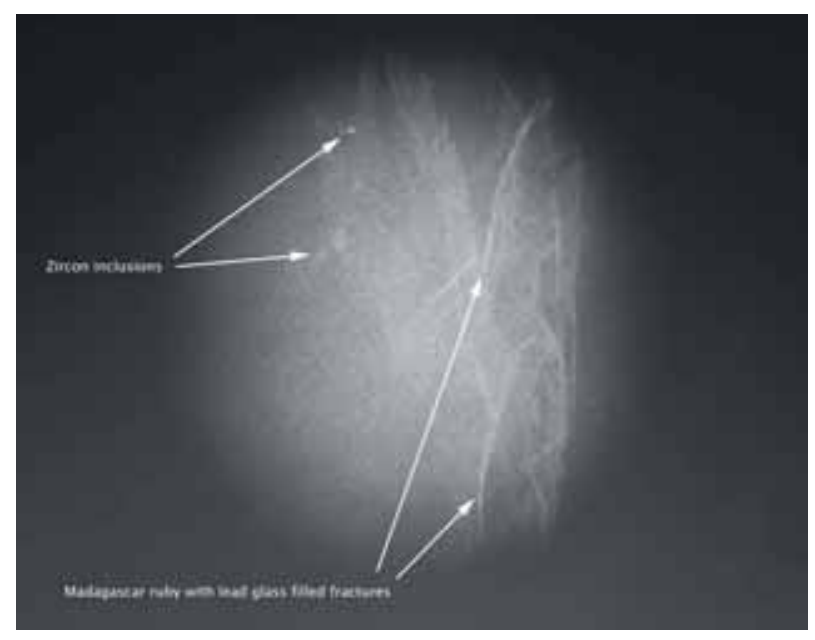

Jewelry Repair Procedures. No damage to the leadglass fillings was observed with standard steam cleaning, ultrasonic cleaning, setting, and even retipping of prongs, when these procedures were performed carefully on the limited number of stones tested, as described below. Some damage was seen with immersion in a pickling solution (figure 23) and exposure to other reagents. The specific tests and results are described below.

Steam Cleaning. Two rubies were selected for steam cleaning. A standard steam cleaner was used, maintaining an approximate pressure of 40-50 psi. Each stone was held in a pair of tweezers in the steam at a distance of approximately one inch for 30 seconds, allowed to cool, and examined in the microscope for damage. This procedure was repeated 15 times for each stone. No damage to the filler was detected in either stone.

Figure 20. At $700^{\circ} \mathrm{C}$, minor amounts of the lead-glass filler began sweating out of the fractures and beading on the surface. Photomicrograph by C. P. Smith; magnified $50 \times$.

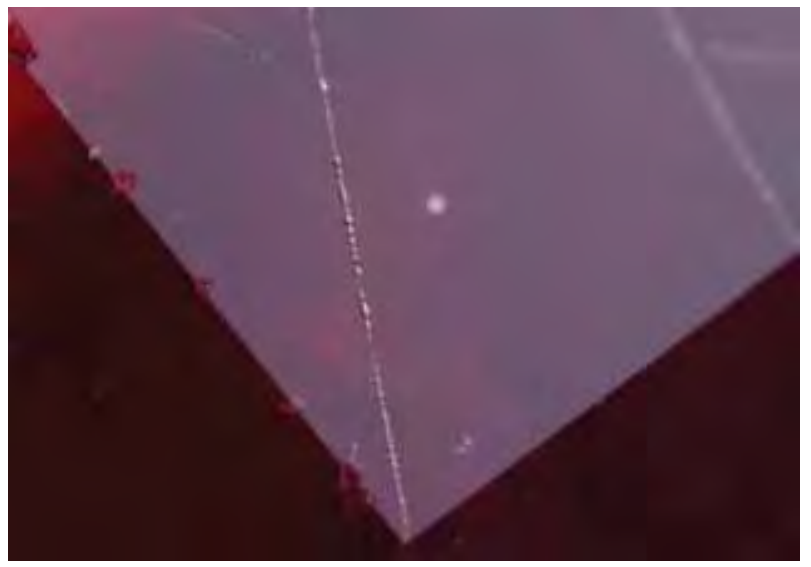




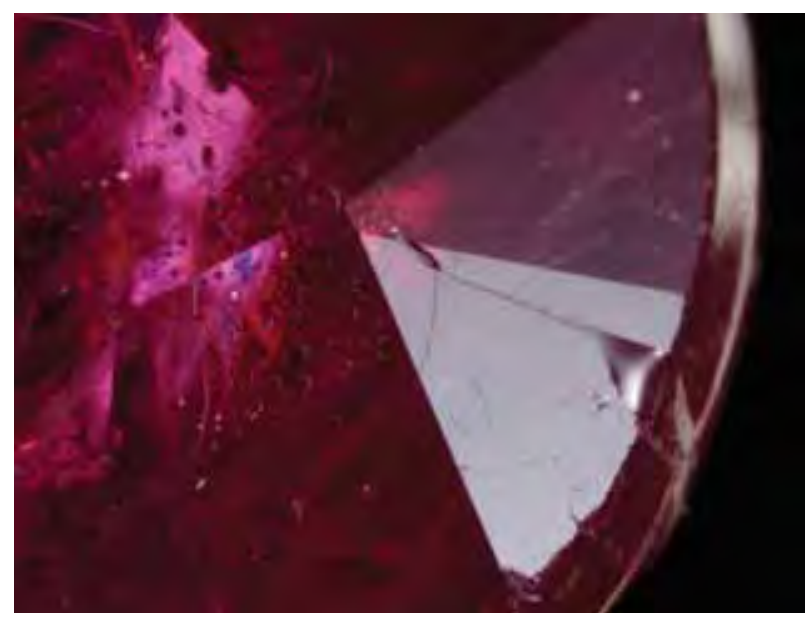

Figure 21. Heating at $700^{\circ} \mathrm{C}$ for only a few minutes caused the lead-based glass in larger cavities to start to flow. Photomicrograph by C. P. Smith; magnified 24x.

Ultrasonic Cleaning. Two different rubies were selected for ultrasonic cleaning. The ultrasonic bath was filled with a standard soap solution that was warmed slightly. Both stones were placed in a wire basket and suspended in the solution with the cleaner turned on. The stones were removed after $15 \mathrm{~min}$ utes and checked for damage; then they were returned to the ultrasonic for another 15 minutes and checked again. Subsequent additional runs (and rechecks) of 30 minutes, 60 minutes, and another 60 minutes were performed for a cumulative total of three hours in the cleaner. No damage to the filler was observed in either stone.

Setting. Four different rubies were set in standard $14 \mathrm{~K}$ yellow gold mountings, the process consisting of mounting the stone, filing the prongs, and polishing the setting. Since lead glasses tend to be relatively soft, we examined the stones carefully after these seemingly benign procedures. No damage to the filler was observed in any stone.

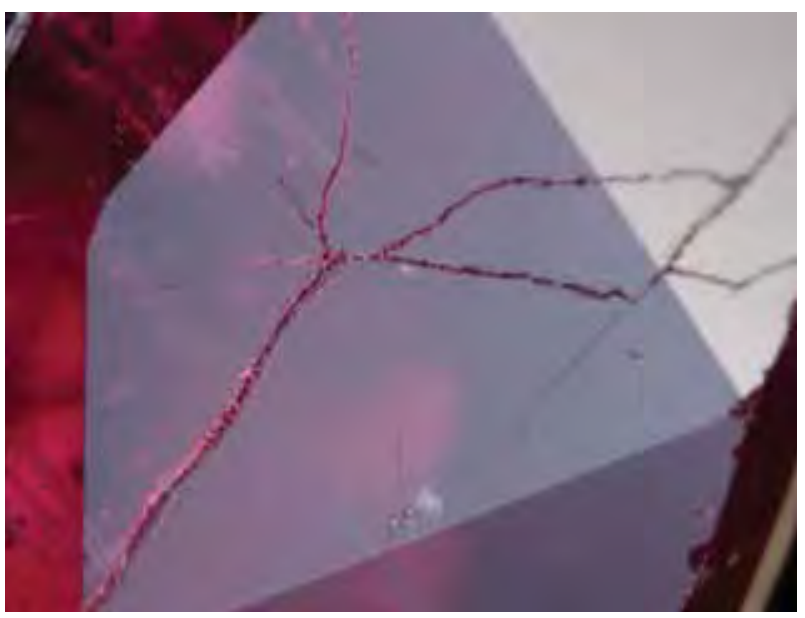

Figure 22. At $800^{\circ} \mathrm{C}$, the lead-glass filler actually started to bubble where fractures reached the surface. Photomicrograph by C. P. Smith; magnified 22x.

Retipping of Prongs. Although jewelers' guidelines typically advise against exposing any ruby or sapphire to the direct heat of a torch during the retipping of prongs, many jewelers routinely perform this procedure with the stone in place. To test the resistance of the filler to standard retipping procedures, we enlisted the aid of two of GIA's Jewelry Manufacturing Arts instructors, Mark Maxwell and Adam Kelley, and began with one of the previously mounted stones. We started with retipping because the temperatures the stones are exposed to during this process are much higher than those for other repair procedures such as sizing. If the filler did not survive the higher-temperature procedure, we would move to one that required lower temperatures to determine the lowest point at which the filler would be damaged.

After some discussion with the instructors, it was decided that they would first perform a retipping procedure that exposed the stone to the lowest temperature possible, assuming the procedure was done correctly. If there was no damage with that
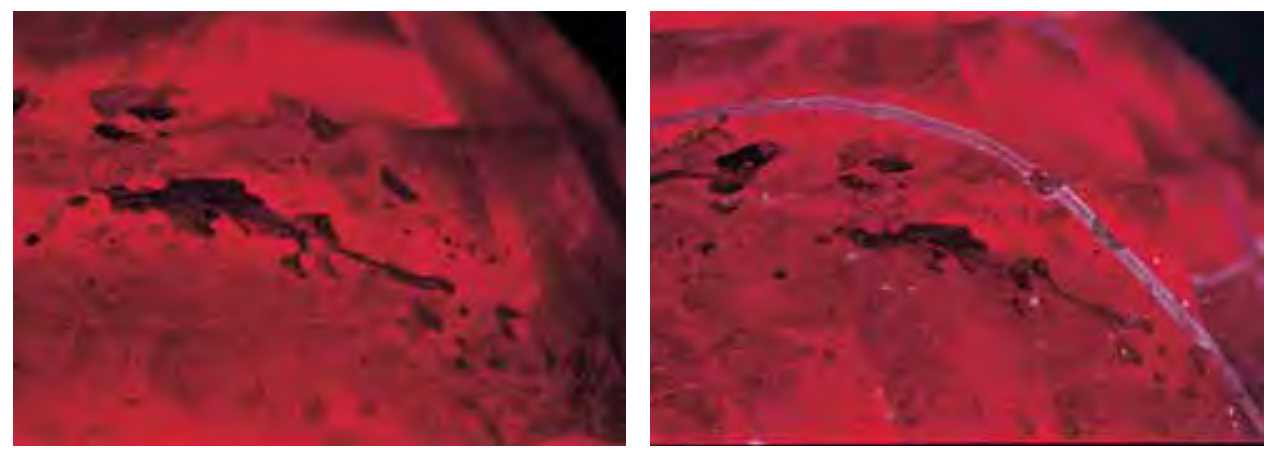

Figure 23. The large fracture in this lead glass-filled ruby is hard to see (left), except for the straight edges on the two flattened gas bubbles in the top center. After immersion in a standard pickling solution for 20 minutes (right), etching of the filler made the edges of the fracture clearly visible. Photomicrographs by S. F. McClure; magnified $30 \times$. 

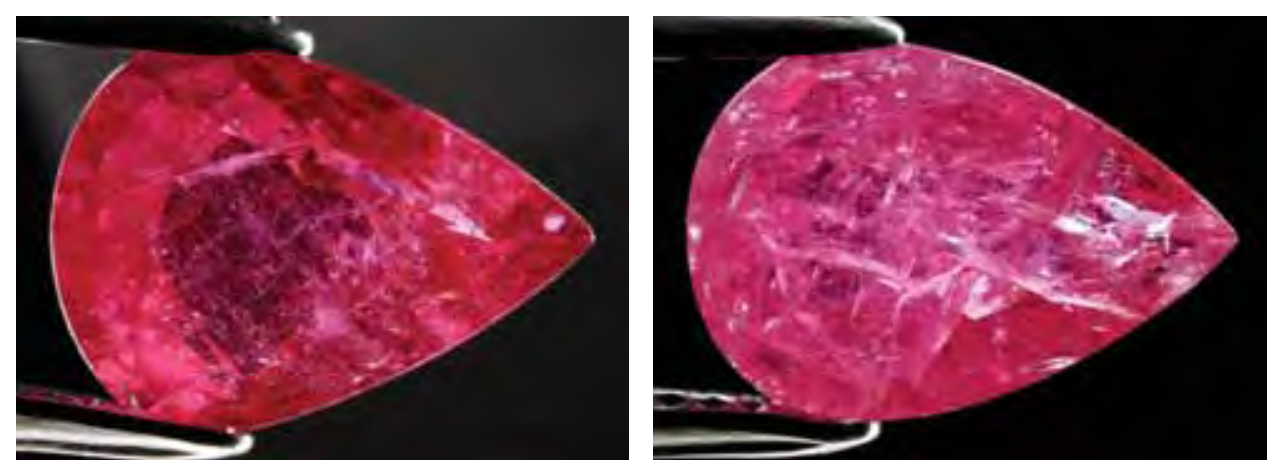

Figure 24. Caustic soda was used to see how much damage a stronger base solutionwould inflict on the glass.

The image on the left shows the 2.08 ct lead glass-filled ruby before the test began; after one hour of exposure (right), the damage to the filler was significant. Photomicrographs by C. P. Smith.

method, we would move to a procedure (on a different stone) that would require the maximum temperature to retip a prong.

The first method involved soldering a piece of $14 \mathrm{~K}$ wire to the prong to be repaired with a "soft" solder, which would then be filed down to rebuild the prong. After the soldering procedure, the mounted stone was immersed in a warm standard pickling solution for one minute. The stone, which had been carefully examined before heating, was examined again. It now showed thin white lines where the fractures reached the surface.

The thought occurred to us that this near-surface damage of the lead-glass filler could be from the heat or the pickling solution, so we repeated the experiment on a second stone and examined it with the microscope immediately after the soldering procedure without placing it in the pickling solution. This time there was no visible damage to the filler. This indicated that the earlier damage was related to the pickling solution, the testing of which will be outlined below.

Next, a higher-temperature procedure was performed on another sample. This retipping process involved soldering a ball of $14 \mathrm{~K}$ gold to the prong. This requires heating of the prong, and therefore the ruby, to a much higher temperature than the earlier procedure. Again the stone was examined (without putting it in pickling solution) and was found to have suffered no damage.

Immersion in Pickling Solution. The second lead glass-filled stone that was subjected to the lowertemperature retipping procedure was placed in the warm pickling solution for one minute to duplicate the first incident. Again, on reexamination we noted a thin ribbon-like area of damage at the point where the filled fractures reached the surface.

This result established a connection between the pickling solution and the damaged filler. Two new unmounted stones with lead-glass filler were chosen and then placed in the pickling solution for 5 and 20 minutes respectively. On reinspection, both stones showed near-surface damage (see, e.g., figure 23), with the depth corresponding directly to the duration of immersion. It was now clear that the caustic pickling solution was etching the lead-glass filler, which made the fractures far more visible.

Exposure to Other Corrosive Solutions. The relatively aggressive solutions, caustic soda and aqua regia, had an immediate reaction with the glasses, etching them readily. In fact, the glass was etched/removed from all the cavities and to a relatively shallow depth in all the fractures after only a few minutes of exposure. However, extending this exposure to 4 hours had little further impact on the removal of the filler. After these tests were performed, the stones looked dramatically different from their appearance prior to testing (see, e.g., figure 24). Nevertheless, because the glass was removed only to a limited depth, their overall appearance was still better than what it would have been if the stone was in its untreated state.

Exposure to Household Products. We also decided to test various products that the stones might encounter once they were purchased by an end-consumer. We were very surprised to find that the aerosol oven cleaner had a similar, very aggressive reaction with the glass, readily etching it. In addition, the ammonia, bleach, and even concentrated lemon juice also had an effect, if less dramatic. In these instances, typically the glass was etched at the very surface of the stones, with the traverses of the fractures newly visible as criss-crossing lines (figure 25).

\section{DISCUSSION AND CONCLUSION}

The significance of any new treatment typically revolves around two points: (1) how effective it is, and (2) whether it can be detected-especially using routine gem testing equipment. As with any treat- 
ment, clarity enhancement by lead-glass filling must be disclosed at all levels of sale to protect consumer confidence.

In the case of lead-glass filling in rubies, the intent is clearly to enhance the apparent clarity of the stone. The treatment is very effective in this regard. Stones that are almost opaque can be improved to the point of being semitransparent to transparent. This makes it possible to market a great deal of previously unusable material.

Fortunately, this treatment is easily detected with magnification. The identifying characteristics are similar to those for diamonds that have been clarity enhanced with a glass filling: very low-relief fractures, gas bubbles and voids (unfilled areas) in fractures, and a blue and orange flash effect. There is also the possibility of a light pink or yellow color to the filler in areas where it is very thick, such as large cavities. However, cavities filled with lead glass are more difficult to detect than those filled with silica glass, so careful observation is required in all cases.

A third concern of consumers and the industry alike is the durability of the treatment to jewelry manufacturing and repair procedures, as well as under normal conditions of wear and care. The lead based-glass filler in the stones we tested turned out to be fairly durable to heat exposure during jewelry repair procedures. However, only three stones were tested and the instructors who performed these procedures for us were highly skilled jewelers. As the controlled heating experiments indicated, too much heat with a torch could still damage this filler, so it would be prudent to unmount stones treated in this manner to be safe.

The filler reacted readily with solvents, particularly with a common jeweler's pickling solution, so these stones should not be exposed to such solutions under any circumstances. Not only does this make it more important to remove such stones from their settings before repair procedures, but it also means that consumers should be informed that damage could occur if the stone is exposed to some common household chemicals.

An interesting debate took place among laboratories as a result of this treatment. The type of starting material used frequently will not display conclusive evidence of heating. The inevitable question then arose: Is the presence of lead-glass filler in fractures sufficient proof that the stone has been subjected to enough heat to say it is heat treated? Our experiments showed that, at least in the samples we obtained, temperatures of at least $700^{\circ} \mathrm{C}$ were neces-

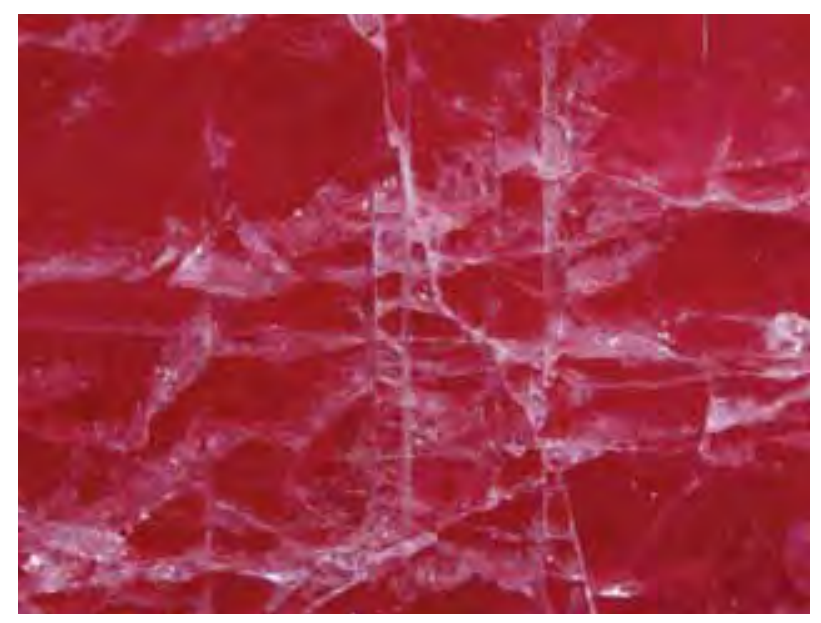

Figure 25. Ammonia, bleach, and even concentrated lemon juice damaged the lead-glass filler at the very surface, producing the whitish areas shown here. Photomicrograph by C. P. Smith; magnified 32x.

sary to soften the glass. For it to flow into fractures, the temperature would obviously have to be much higher $-800^{\circ}$ or $900^{\circ} \mathrm{C}$ at least. We know that these temperatures are high enough to facilitate changes in corundum, particularly in relation to color (Nassau, 1994). It has been hypothesized that formulations of lead glasses could be developed that would have much lower melting points, but as we have not yet observed such glasses ourselves, we maintain that the presence of this glass in fractures is sufficient proof to say the stone also has been heat treated.

What is perhaps most remarkable about this treatment is the type of material it brings to the market. High-temperature heat treatment and healing of fractures brought Mong Hsu ruby to the market years ago for what was at the time a very low price. Clarity enhancement with high-lead-content glass has brought ruby and pink sapphire to the market for even lower prices. Of course, the color of the low end of this material is not ideal, but some of the samples acquired for this study were only $\$ 2.00$ per caratstones over $1 \mathrm{ct}$ in size that were mostly transparent face up and ranged from purplish red to purplish pink. Inexpensive synthetics cost more than this. We purchased the 2.31 lead glass-filled ruby in figure 5 at the 2006 Tucson shows for \$20 per carat. Certainly, better-quality stones-also represented as lead-glass filled, as these were-are being sold for significantly higher prices, but we believe the low end is a new low for treated natural ruby.

From the moment we saw this treatment, we believed it would eventually be used for much higherquality goods. The efficiency of the treatment is such 
that a single large fracture in an otherwise clean ruby could be made to "disappear" to the unaided eye, exactly as filled fractures can be made to "disappear" in emeralds and diamonds. In fact, we have already seen several stones that fall into this category.

With glass being such a versatile material, other formulations may be possible, which could change the properties of the filler, including its melting point. The properties we reported here are only those we observed in our study samples. Last year, two laboratories (AGTA and GAAJ) reported seeing a lead-glass filler in star rubies that did not exhibit a flash effect (Befi and DuToit, 2005; Kitawaki et al., 2005). While no quantitative chemical data was given in either report, it is clear from the lower surface luster of the filler in the images provided that the R.I.-and therefore the composition - of the filler discussed in those reports must be different from the lead-glass fillers we studied. Thus, it is probable there are stones on the market that have been treated in a similar fashion with a glass of different composition. It is imperative that laboratories worldwide continue to monitor the material reaching the market, so that if and when a change occurs that might alter the identifying characteristics of the stones, the greater gemological community can be made aware of it as soon as possible.
ABOUT THE AUTHORS

$\mathrm{Mr}$. McClure is director of identification services at the GIA Laboratory in Carlsbad. Mr. Smith is director of identification services, Dr. Wang is research scientist, and Mr. Hall is manager of analytical research, at the GIA Laboratory in New York

ACKNOWLEDGMENTS: The authors thank Paul Wild, Colorline, and Harpaz Gem \& Pearl Inc. for graciously providing samples. They are grateful to Dr. Yingwei Fei of the Carnegie Institute of Washington for arranging the SEM-
WDS analysis. Mark Maxwell and Adam Kelley, Jewelry Manufacturing Arts instructors in the GIA Education department, provided advice and assistance by retipping the prongs of some of the sample rubies. The authors acknowledge with appreciation David Kondo, Kyaw Soe Moe, Paul Johnson, Wendi Mayerson, and Carolyn van der Bogert (all of the GIA Laboratory New York Identification Department), as well as Mike Breeding, Andy Shen, and Sam Muhlmeister (of the GIA Laboratory Carlsbad Research Department) and Ken Scarratt (of the GIA Research Laboratory Bangkok) for analyses of the samples and many fruitful discussions.

\section{REFERENCES}

AGTA Gemological Testing Center (2004) New ruby treatment arrives in the United States. www.agta-gtc.org/2004-07-02 rubytreatment.htm, July 2.

AGTA Gemological Testing Center (2005) Lead glass fracture filling in ruby. www.agta-gtc.org/2005-01-06_glassfilledruby.htm, Jan. 6.

Befi R., Dutoit G. (2005) Glass-filled star ruby. www.agta-gtc.org/ 2005-09-20_glass-filled_star_ruby.htm, Sept. 20.

Emmett J.L., Scarratt K., McClure S.F., Moses T., Douthit T.R., Hughes R., Novak S., Shigley J.E., Wang W., Bordelon O., Kane R.E. (2003) Beryllium diffusion of ruby and sapphire. Gems $\oplus$ ) Gemology, Vol. 39, No. 2, pp. 84-135.

GAAJ Research Laboratory (2004) Lead-glass impregnated ruby. www.gaaj-zenhokyo.co.jp/researchroom/kanbetu/2004/ gaaj_alert-040315en.html, Mar. 15.

Kane R.E. (1984) Natural rubies with glass-filled cavities. Gems Gemology, Vol. 20, No. 4, pp. 187-199.

Kitawaki H., Abduriyim A., Okano M. (2005) Lead glass-permeated ruby-Update. Gemmology, Vol. 36, No. 425, pp. 14-15.

Koivula J.I., Kammerling R.C., Fritsch E., Fryer C., Hargett D., Kane R.E. (1989) The characteristics and identification of filled diamonds. Gems \&) Gemology, Vol. 25, No. 2, pp. 68-83.

Li-Jian Q., Zeng C.G., Xin-qiang Y. (2005) Lead-rich glass substance in filled treated rubies. Journal of Gems and Gemmology, Vol. 7, No. 2, pp. 1-6.

McClure S.F., Smith C.P. (2000) Gemstone enhancement and detection in the 1990s. Gems ef Gemology, Vol. 36, No. 4, pp. 336-359.

Milisenda C.C., Horikawa Y., Henn U. (2005) Rubine Mit Bleihaltigen Glasern Gefullt. Gemmologie: Zeitschrift der Deutschen Gemmologishen Gesellschaft, Vol. 54, No. 1, pp. 35-42.

Nassau K. (1994) Gemstone Enhancement: History, Science and the State of the Art, 2nd ed. Butterworth/Heinemann Ltd., Oxford, UK, $127 \mathrm{pp}$.

Pardieu V. (2005) Lead glass filled/repaired rubies. www.aigslaboratory.com/Filearticle/55.pdf, Jan. 17.

Peretti A., Schmetzer K., Bernhardt H-J. (1995) Rubies from Mong Hsu. Gems «) Gemology, Vol. 31, No. 1, pp. 2-27.

Rockwell K.M., Breeding C.M. (2004) Gem Trade Lab Notes: Rubies, clarity enhanced with a lead glass filler. Gems «) Gemology, Vol. 40, No. 3, pp. 247-249.

Smith C.P., McClure S.F., Wang W., Hall M. (2005) Some characteristics of lead-glass filled corundum. Jewellery News Asia, No. 255 , pp. 79, 82-84.

SSEF Swiss Gemmological Institute (2005) Lead glass filled fractures in rubies. Facette, No. 12, p. 10, www.ssef.ch/facette12.pdf.

Sturman N. (2005) Gem News: Lead glass-filled rubies appear in the Middle East. Gems et Gemology, Vol. 41, No. 2, pp. 184-185.

Themelis T. (2005) Glass-filled rubies. Australian Gemmologist, Vol. 22, No. 8, pp. 360-365. 